\title{
DARAPLADIB INHIBIT ADHESION MOLECULE EXPRESSION IN AORTA AT EARLY STAGES OF ATHEROSCLEROSIS USING SPRAGUE-DAWLEY TYPE 2 DIABETES MELLITUS MODEL
}

\author{
HERIANSYAH $\mathrm{T}^{1}$, HANIFA $\mathrm{H}^{2}$, ANDARINI $\mathrm{S}^{2}$, WIHASTUTI TITIN ANDRI ${ }^{2 *}$ \\ ${ }^{1}$ Department of Cardiology, Syiah Kuala University, Aceh, Sumatera, Indonesia. ${ }^{2}$ Department of Biomedical, Brawijaya University, Malang, \\ East Java, Indonesia. Email: titinwihastuti@gmail.com
}

Received: 10 February 2017, Revised and Accepted: 09 March 2017

\section{ABSTRACT}

Objective: Hyperglycemia and hyperlipidemia in diabetes mellitus (DM) can lead an atherosclerosis. The increase of low-density lipoprotein level in DM and atherosclerosis is correlated with lipoprotein-associated phospholipase $A_{2}$ (Lp-PLA 2 ). Lp-PLA is an enzyme that produces lysophosphatidylcholine (LysoPC) and oxidized nonesterified fatty acids. LysoPC regulated inflammation mediators, include cytokines, adhesion molecules (such as vascular cell adhesion molecule-1 [VCAM-1] and intercellular adhesion molecules-1 [ICAM-1]), and monocyte chemoattractant protein-1 (MCP-1) chemotactic. Darapladib is known as a Lp-PLA 2 specific inhibitor. It is also considered to be an atherosclerosis treatment. The aim of this study is to know darapladib effect on VCAM-1 and ICAM-1 aorta expression in early stages of atherosclerosis using Sprague-Dawley Type 2 DM (T2DM) model.

Methods: About 30 Spraque-Dawley rats are divided into three main groups: Normal, T2DM, and T2DM with darapladib administration group. Each group consists of 2 serials treatment time: 8 and 16 weeks treatment group. Fasting blood glucose, resistance insulin, and lipid profile were measured and analyzed to ensure T2DM model. VCAM-1 and ICAM-1 expression were measured using double staining immunofluorescence. Each data were analyzed using one-way ANOVA.

Results: There is a significant difference in VCAM- 1 expression in T2DM group ( 8 and 16 weeks), with $\mathrm{p}=0.011$ and 0.034 ( $<<0.05$ ), respectively. Mean while, a significant difference for ICAM- 1 only showed in 8 weeks T2DM group with $\mathrm{p}=0.03(\mathrm{p}<0.05)$. Moreover, there is a decreasing trend in 16 weeks T2DM group.

Conclusion: Our results showed that darapladib can decrease VCAM-1 and ICAM-1 aorta expression in early stages of atherosclerosis using SpragueDawley T2DM model. This showed another evidence of darapladib as atherosclerosis treatment.

Keywords: Darapladib, Vascular cell adhesion molecule-1, Intercellular adhesion molecules-1, Atherosclerosis, Type 2 diabetes mellitus.

(c) 2017 The Authors. Published by Innovare Academic Sciences Pvt Ltd. This is an open access article under the CC BY license (http://creativecommons. org/licenses/by/4. 0/) DOI: http://dx.doi.org/10.22159/ajpcr.2017.v10i6.17631

\section{INTRODUCTION}

Diabetes mellitus (DM) is a group of metabolic diseases characterized by hyperglycemia as a result of a decrease in insulin, insulin action, or of both [1]. International Diabetes Federation (2013) state that there are about 382 million adults or about $8.3 \%$ who had diabetes, and this number is expected to increase until 592 million in $<25$ years [2]. The high prevalence of diabetes is expected to increase atherosclerosis prevalence [3]. This is because DM is a major risk factor for atherosclerosis development and clinical manifestations, including coronary artery disease, stroke, and peripheral vascular disease [4].

Hyperglycemia induces most of the changes at the cellular level in vascular tissue that potentially accelerate atherosclerosis process [5]. Atherosclerosis is influenced by cellular adhesion molecules (CAM) interaction [6]. High low-density lipoprotein (LDL) level in atherosclerosis can increase free radical that vulnerable to various oxidative process and triggered proinflammatory cells recruitment and adherence [7]. Atherosclerosis affects a discrete portion of the blood vessels, such as vessel curvature and bifurcation. These sections are marked by disturbed oscillatory flow which induces the upregulation of adhesion molecules pro-inflammatory, such as inter CAM (ICAM)-1 and vascular cell adhesion molecule (VCAM)-1. Chemokines will stimulate leukocytes and upregulate $\alpha 4$ and $\beta 2$ integrin binding to VCAM-1 and ICAM-1, which will mediate the next stage [8].

LDL, oxidized LDL (oxLDL), and free fatty acid level were increased in T2DM (T2DM) [9]. LDL cholesterol is harmful because of lipid peroxidation process (auto-oxidation) [7]. LDL is closely related with enzyme lipoprotein-associated phospholipase $A_{2}$ (Lp-PLA $A_{2}$. This enzyme was originally known as platelet-activating factoracetylhydrolase (PAF-AH), which had two biological activities such as inactivate PAF-AH pro-inflammatory mediators and hydrolyze oxidative modified polyunsaturated fatty acids that produce lysophosphatidylcholine (LysoPC) and oxidized nonesterified fatty acids (OxNEFA). OxNEFA have chemotactic activity of monocytes, and LysoPC is regulating inflammatory mediators, including cytokines, adhesion molecules, and MCP-1 chemotactic mediator [10].

Darapladib was known as a specific inhibitor of Lp-PLA ${ }_{2}$ and already developed as a drug for atherosclerosis [11]. Until now a Lp-PLA 2 role in atherogenesis still ambiguous [12]. There are few studies of darapladib effects in atherosclerosis. One of them is the research conducted by Heriansyah et al. It showed that darapladib administration can reduce ox-LDL and foam cell amount [4].

Therefore, the aim of this study is to know darapladib effect on VCAM-1 and ICAM-1 aorta expression in early stages of atherosclerosis using Sprague-Dawley T2DM model. In this way, the result of this research can be useful for darapladib evidence as atherosclerosis drug because it is still controversial whether darapladib has a positive or negative effect.

\section{METHODS}

Study groups

This study used 4 weeks male Sprague-Dawley and weight around 150-200 g. Samples were obtained from Bogor Agricultural University, Bogor, Indonesia. These rats were divided into three main groups: 
Normal, T2DM, and T2DM with darapladib administration group. Each group consists of 2 serials treatment time: 8 and 16 weeks. T2DM model groups (T2DM) which fed with high-fat diet and injected with streptozotocin (STZ) intraperitoneal low dose $35 \mathrm{mg} / \mathrm{kgBW}$. Darapladib was obtained from GlaxosmithKline. Samples were given darapladib orally $20 \mathrm{mg} / \mathrm{kgBW}$ once a day according to the time serial groups given. Normal rats food contained $3.43 \mathrm{kcal} / \mathrm{g}$ total energy calories, while the HFD contained $5.29 \mathrm{kcal} / \mathrm{g}$ total calorie energy. $30 \mathrm{~g}$ of food were given for each rat everyday. Parameters measurement was done at the Central Laboratory of Biological Sciences, Brawijaya University.

\section{Measurement to ensure T2DM model}

\section{Blood glucose measurement}

STZ $20 \mathrm{mg} / \mathrm{kgBW}$ was administered before blood glucose first measurement to induce T2DM. T2DM was diagnosed after blood glucose level measurement using GlucoDR blood glucose test meter (All Medicus Co., Ltd., Dongan-gu, Anyang-si, Korea). T2DM was diagnosed after obtaining fasting blood glucose levels $>126 \mathrm{mg} / \mathrm{dl}$ in rats.

\section{Insulin resistance measurement}

Insulin in rat's blood plasma was measured using Rat INS (insulin) ELISA kit (Cat. No. E-EL-R2466). The obtained results are still in $\mathrm{ng} / \mathrm{mL}$ units. Plasma insulin levels were converted into IU/L. WHO formula was used by dividing the result with 0.0347 , as 1 IU is equivalent with $0.0347 \mathrm{mg} / \mathrm{L}$ [13]. Insulin resistance can be measured with homeostatic model assessment-insulin resistance (HOMA-IR) formula especially in rats, which required some data, such as fasting glucose and plasma insulin levels by the following formula [14]:

$$
\text { HOMA }- \text { IR }=\frac{\text { FBSxFINS }}{14.1}
$$

Explanation:

HOMA-IR: Homeostatic model assessment-insulin resistance FBG: Fasting blood glucose $(\mathrm{mmol} / \mathrm{L})$

FINS: Fasting insulin plasma (microU/L)

Interpretation of HOMA-IR calculation in rats is if the result $>1.716$ then it can be categorized as insulin resistance with $83.87 \%$ sensitivity and $80.56 \%$ specificity ( $95 \%$ confidence interval) [15].

\section{Lipid profile measurement}

Lipid profiles total cholesterol, high-density lipoprotein (HDL), and LDL were measured in rat blood serum using EnzyChrom ${ }^{\mathrm{TM}}$ kit. $^{2}$

\section{VCAM-1 AND ICAM-1 EXPRESSION MEASUREMENT USING DOUBLE STAINING IMMUNOFLUORESCENCE}

VCAM-1 and ICAM-1 in aortic tissue was measured by immunofluorescence. Aortic tissue was previously fixed with PHEMO buffer (68 mM PIPES, 25 mM, HEPES, pH 6.9, 15 mM EGTA, 3 mM MgCl , $10 \%[\mathrm{v} / \mathrm{v}]$ dimethyl sulfoxide containing $3.7 \%$ formaldehyde, and $0.05 \%$ glutaraldehyde) and were processed by immunofluorescence labeling with anti-rat antibody ICAM-1 using rhodamin secondary antibody (BIOS Inc., Boston, MA, USA) and anti-rabbit antibody VCAM-1 using FITC secondary antibody (Santacruz, USA). This parameter was observed with confocal laser scanning microscopy (Olympus Corporation, Tokyo, Japan) and was quantitatively analyzed using Olympus FluoView software (version 1.7A; Olympus Corporation).

\section{Statistical analysis}

Blood glucose, insulin resistance, lipid profile, VCAM-1, and ICAM-1 expression were documented, and normality test was performed to determine the normality of data. Data were analyzed using one-way ANOVA and continue with Duncan multiple range test to compare each groups (more than two groups). All the analysis was performed using SPSS statistical software, version 16.0 and $\mathrm{p}<0.05$ was considered to be statistically significant.

\section{Animal ethical approval}

Ethical approval was obtained for the animal treatment and experimental processes in this study from the Animal Care and Use Committee Brawijaya University Number 400/EC/KEPK/10/2016.

\section{RESULTS}

Fasting blood glucose, insulin resistance, and lipid profile were measured to ensure T2DM model in Sprague-Dawley rats. T2DM model was made successfully if fasting blood glucose $>126 \mathrm{mg} / \mathrm{dl}$ and insulin resistance (using HOMA-IR) results $>1.716$ (Table 1). Table 1 shows that there is a significance different in lipid profile (cholesterol total, HDL, and LDL) between normal and DM group. This result shows that Sprague-Dawley T2DM model was successfully made.

From the conducted research, the average of VCAM-1 expression was ranged from 611.1402 to $999.0005 \mathrm{pg} / \mathrm{ml}$. The lowest VCAM-1 expression was normal 8 weeks group $(611.1402 \mathrm{pg} / \mathrm{ml})$, compared with the other groups (normal 16 weeks, 8 weeks DM, DM 16 weeks, Duchenne muscular dystrophy patients (DMDP) 8 weeks, and 16 weeks DMDP). For normal 16 weeks group, VCAM-1 expression $(768.1375 \mathrm{pg} / \mathrm{mI})$ was higher than DMDP groups ( 8 and 16 weeks). The highest expression of VCAM-1 (999.0005 AU) was DM 16 weeks group (Table 1).

There are significant differences in the expression of VCAM-1 between DM and DMDP group. The expression of VCAM-1 in DM 8 weeks is 911.8187 and VCAM-1 expression in DMDP 8 weeks is 596.2806, with $\mathrm{p}=0.011(\mathrm{p}<0.05)$. While the expression of VCAM- 1 in DM 16 weeks is 999.0005 and the average expression of VCAM-1 on DMDP 16 weeks is 684.7586, with $\mathrm{p}=0.034(\mathrm{p}<0.05)$.

Meanwhile, the average expression of ICAM-1 was observed from 442.5055 to $1010,727 \mathrm{pg} / \mathrm{ml}$. The lowest ICAM-1 expression was DMDP 8 weeks group (442.5055). For normal 16 weeks group, ICAM I expression (542.0247) is lower than DMDP groups (8 and 16 weeks). The highest ICAM-1 expression (1010.727) was DM 16 weeks group (Table 1).

This study showed a significant decrease in ICAM-1 expression between DM 8 weeks group and DMDP 8 weeks group with an average ICAM-1 expression 991.274 and 573.9291, respectively, with $\mathrm{p}=0.03$ ( $\mathrm{p}<0.05)$. Meanwhile, there is no significant difference in DM 16 weeks and DMDP 16 weeks group, although showed a decreasing trend, with the average of ICAM-1 expression is 1010.727 and 874.8462 , respectively, with $\mathrm{p}=0.884(\mathrm{p}<0.05)$. An example for VCAM- 1 and ICAM-1 expression using immunofluorescence can be seen in Fig. 1.

\section{DISCUSSION}

Darapladib is a carbon-based small molecules that are taken orally which specifically inhibits Lp-PLA 2 at certain doses. Inhibition of

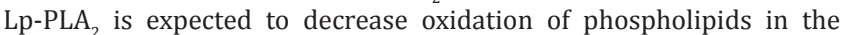
vascular space and will reduce adhesion molecules expression and
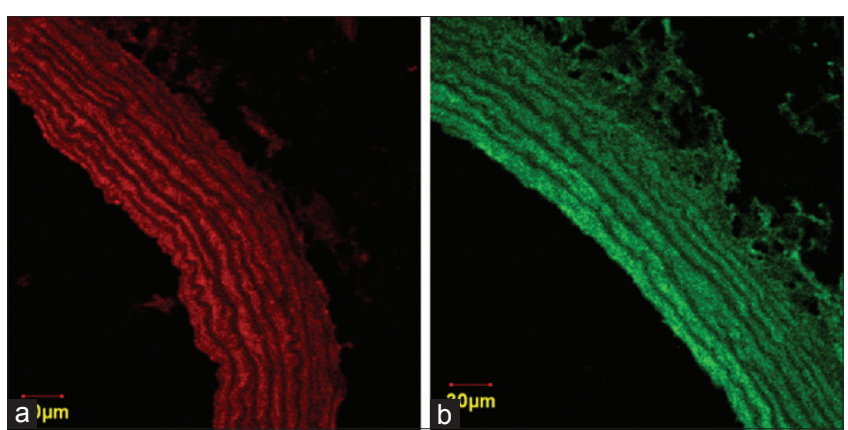

Fig. 1: (a) Intercellular cell adhesion molecules-1 expression using rhodamin secondary antibody and (b) Vascular cell adhesion molecule-1 expression using FITC secondary antibody 


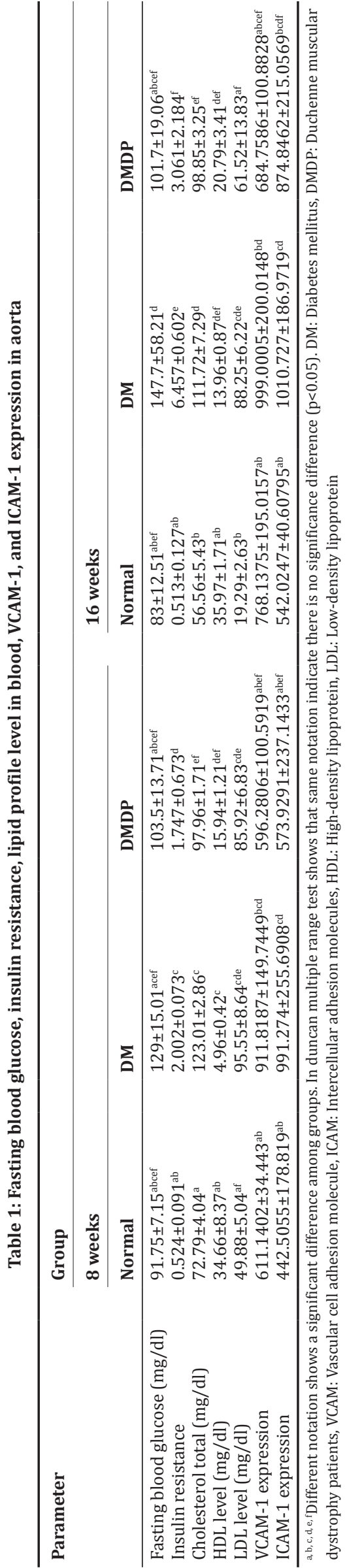

other factors that lead to atherosclerosis. Darapladib has been shown to reduce the content of LysoPC and the expression of 24 genes related with macrophages and $\mathrm{T}$ lymphocytes function, with a decrease in plaque and necrotic areas [16]

Based on research conducted by Wang et al. in 2011 with the title inhibition of Lp-PLA ${ }_{2}$ ameliorates and decreases inflammation of atherosclerotic plaque formation in ApoE-deficient mice, showed expression of VCAM-1 and ICAM-1 were lower in the group darapladib [17]. This is consistent with the results in this study that showed significant differences in VCAM-1 expression between the DM group with DMDP.

The decreased expression of VCAM-1 in darapladib group could be due to the darapladib action as a Lp- $\mathrm{PLA}_{2}$ specific inhibitor. This action causing a decrease of LysoPC, Lp-PLA product, which induces a wide variety of inflammatory gene expression. LysoPC can stimulate the expression of VCAM-1/ICAM-1 and MCP-1 in endothelial cells or vascular smooth muscle cell [17]. With the Lp-PLA ${ }_{2}$ inhibition may decrease LysoPC and ultimately reduce the expression of VCAM-1.

VCAM-1 known to have an important role in atherosclerosis. This is due to tumor necrosis factor- $\alpha$ (TNF- $\alpha$ ) signaling pathways. TNF- $\alpha$ has been known to have an important role in the initiation of inflammation [18]. TNF- $\alpha$ signaling pathways mediated by nuclear factor-kappa-B and responsible for adhesion molecules expression such as VCAM-1 and ICAM-1 in the endothelium. TNF- $\alpha$ is known to induce the expression of ICAM-1, likely through the protein kinase C (PKC)- $\delta$. The adhesion molecules allow leukocytes are sticking to endothelium and their transmigration into peripheral tissues [19]. An increased expression of membrane-bound VCAM-1 can reflect atherosclerotic lesion formation progression [20].

In their research, Sumagin et al. examined the role of ICAM-1 and the possible role of leukocyte-endothelial interactions also the regulation of the vascular permeability. During inflammation development, there are two major events. Leukocytes will interact with endothelium in the process that will lead to the cells passing through the barrier created by the endothelial cells. Migration of these cells will lead to vascular permeability modification which causes a transfer of solutes into peripheral tissues. Sumagin et al. showed that both arterioles and venules can respond pro-inflammatory stimuli. Under basal conditions, changes in vascular permeability associated with the expression of ICAM-1 on PKC [21].

In this study showed a significant increase in adhesion molecule between normal and DM group. This may be caused by hyperglycemia in DM. Hyperglycemia can enhance monocyte adhesion to endothelial cells [22]. Meanwhile, in this study shows a decrease in ICAM-1 expression between DM 8 weeks and DMDP 8 weeks groups with $\mathrm{p}=0.03(\mathrm{p}<0.05)$. While there was no significant decrease in DM 16 weeks and DMDP 16 weeks group, although showed a decreasing trend $(\mathrm{p}<0.05)$. Decreased expression of ICAM-1 on darapladib administration is still controversial. Research conducted by Hu et al. in 2011 showed that darapladib only decreased the expression of VCAM-1 but do not decrease the expression of ICAM-1 [23]. Meanwhile, research conducted by Wang et al. in 2011 showed that the expression of ICAM-1 in the darapladib group is lower than the positive control group [17], which is same with this study.

\section{CONCLUSION}

Our results study showed that darapladib can decrease VCAM-1 and ICAM-1 aorta expression in early stages of atherosclerosis using Sprague-Dawley T2DM model. This showed another evidence of darapladib as atherosclerosis treatment.

\section{REFERENCES}

1. American Diabetes Association. Diagnosis and classification of diabetes mellitus. Diabetes Care 2011;34(1):S62. 
2. IDF Atlas. IDF Diabetes Atlas. $6^{\text {th }}$ ed. Brussels: IDF; 2013. p. 9.

3. Chait A, Bornfeldt KE. Diabetes and atherosclerosis: Is there a role for hyperglycemia? J Lipid Res 2009;50 Suppl: S335-9.

4. Heriansyah T, Wihastuti TA, Anita KW, Iskandar A, Suhendra RB, Setiabudi PA, et al. Atherogenesis inhibition by darapladib administration in dyslipidemia model sprague-dawley rats. Natl $\mathrm{J}$ Physiol Pharm Pharmacol 2015;2:2.

5. Aronson D, Rayfield EJ. How hyperglycemia promotes atherosclerosis: Molecular mechanisms. Cardiovasc Diabetol 2002;1:1.

6. Galkina E, Ley K. Vascular adhesion molecules in atherosclerosis. Arterioscler Thromb Vasc Biol 2007;27(11):2292-301.

7. Wihastuti TA, Sargowo D, Tjokroprawiro A, Permatasari N, Widodo MA, Soeharto S. Vasa vasorum anti-angiogenesis through H2O2, HIF-1a, NF-?B, and iNOS inhibition by mangosteen pericarp ethanolic extract (Garcinia mangostana Linn) in hypercholesteroldiet-given Rattus norvegicus wistar strain. Vasc Health Risk Manag 2014; 10:523-31

8. Wihastuti TA, Heriansyah T, Soraya M, Wijayanti M, Firani N, Iskandar A, et al. Inhibition of oxidative stress in hypercolerterolemic rats by soy milk. J Cardiovasc Disease Res 2016;7(2):74-82.

9. Belma H, Dhalla N. Diabetic Cardiomyopathy: Biochemical and Molecular Mechanism. New York: Springer-Verlag; 2014. p. 221

10. Carlquist JF, Muhlestein JB, Anderson JL. Lipoprotein-associated phospholipase A2: A new biomarker for cardiovascular risk assessment and potential therapeutic target. Expert Rev Mol Diagn 2007;7:511-7.

11. Thompson PL, Nidorf SM, Eikelboom J. Targeting the unstable plaque in acute coronary syndromes. Clin Ther 2013;35(8):1099-10.

12. Heriansyah T, Adam AA Wihastuti TA, Rohman MS Elaborate evaluation of serum and tissue oxidized LDL level with darapladib therapy: A feasible diagnostic marker for early atherogenesis. Asian Pac J Trop Biomed 2016. DOI: 10.1016/j.apjtb.2016.11.014.

13. Burns TA, Geor RJ, Mudge MC, McCutcheon LJ, Hinchcliff KW, Belknap JK. Proinflammatory cytokine and chemokine gene expression profiles in subcutaneous and visceral adipose tissue depots of insulinresistant and insulin-sensitive light breed horses. J Vet Intern Med
2010;24(4):932-9.

14. van Dijk JW, Venema M, van Mechelen W, Stehouwer CD, Hartgens F, van Loon LJ. Effect of moderate-intensity exercise versus activities of daily living on 24-hour blood glucose homeostasis in male patients with Type 2 diabetes. Diabetes Care 2013;36(11):3448-53

15. Cacho J, Sevillano J, de Castro J, Herrera E, Ramos MP. Validation of simple indexes to assess insulin sensitivity during pregnancy in wistar and Sprague-Dawley rats. Am J Physiol Endocrinol Metab 2008;295(5):E1269-76

16. Wilensky RL, Shi Y, Mohler ER $3^{\text {rd }}$, Hamamdzic D, Burgert ME, Li J, et al. Inhibition of lipoprotein-associated phospholipase A2 reduces complex coronary atherosclerotic plaque development. Nat Med 2008;14:1059-66

17. Wang WY, Zhang J, Wu WY, Li J, Ma YL, Chen WH, et al. Inhibition of lipoprotein-associated phospholipase A2 ameliorates inflammation and decreases atherosclerotic plaque formation in ApoE-deficient mice. PLoS One 2011;6(8):e23425

18. Sethi G, Sung B, Aggarwal BB. TNF: A master switch for inflammation to cancer. Front Biosci 2008;13:5094-107.

19. Frank PG, Lisanti MP. ICAM-1: Role in inflammation and in the regulation of vascular permeability. Am J Physiol Heart Circ Physiol 2008;295(3):H926-7

20. Subhapriya S, Tomi L, Padmanaban VC. Atherosclerosis: Critical role of oxidation and inflammation. Int J Pharm Pharm Sci 2013:5(4):6-8

21. Sumagin R, Lomakina E, Sarelius IH. Leukocyte-endothelial cell interactions are linked to vascular permeability via ICAM-1-mediated signaling. Am J Physiol Heart Circ Physiol 2008;295(3):H969-77.

22. Navale AM, Paranjape AN. Role of inflammation in development of diabetic complications and commonly used inflammatory markers with respect to diabetic complications. Int J Pharm Pharm Sci 2013;5(2):1-5.

23. Hu MM, Zhang J, Wang WY, Wu WY, Ma YL, Chen WH, et al. The inhibition of lipoprotein-associated phospholipase A2 exerts beneficial effects against atherosclerosisin LDLR-deficient mice. Acta Pharmacol Sin 2011;32:1253-8 